\title{
Fluorescence and UV-VIS Spectroscopy to Determine the Quality Changes of Rapeseed Oil Fortified with New Antioxidant After Storage Under Various Conditions
}

\author{
Dobrochna Rabiej $^{1}$ • Aleksandra Szydłowska-Czerniak ${ }^{1}$ (D)
}

Received: 18 March 2020 / Accepted: 14 June 2020 / Published online: 9 July 2020

(C) The Author(s) 2020

\begin{abstract}
This work focused on the application of synchronous fluorescence (SF) spectroscopy, excitation-emission matrix (EEM) fluorescence spectroscopy and modified UV-VIS methods for characterization of oxidative status and antioxidant activity (AA) of refined rapeseed oils fortified with the novel antioxidant octyl sinapate (OSA) at two concentrations and butylated hydroxyanisole (BHA) stored in different types of packages under various storage conditions. Quality parameters such as amounts of the primary and secondary oxidation products in rapeseed oils increased after storage under various conditions, whereas the AA of oils decreased. However, OSA strongly inhibited the generation of primary oxidation products in oils exposed to light. The AA of rapeseed oil with $0.5 \%$ of OSA packed in transparent glass after exposure to light was 6 times higher than the AA of non-supplemented oil. Moreover, the intensities of bands attributed to tocopherols and phenolic compounds decreased during storage depending on the storage conditions, but characteristic peaks for generation of oxidation products appeared. Interestingly, the fluorescence spectra of oil fortified with $0.5 \%$ of OSA contained the information of a new fluorescent product. Moreover, the potential usefulness of EEM to study the effect of antioxidants on degradation of stored rapeseed oils was demonstrated. Fluorescence spectroscopy can be used to investigate the oxidation state of rapeseed oils packed in various containers during storage at different conditions. The addition of the lipophilic antioxidant to rapeseed oil effectively delayed the oxidation process and significantly increased its AA.
\end{abstract}

Keywords Fluorescence spectroscopy $\cdot$ Quality parameters $\cdot$ Octyl sinapate $\cdot$ Rapeseed oil $\cdot$ Packaging $\cdot$ Storage

\section{Introduction}

Rapeseed oil is the most popular vegetable oil in Poland, while rapeseed is the second largest oilseed crop in the world, after soybean. Rapeseed oil is consider the healthiest culinary oil due to the presence of components such as unsaturated fatty acids with a balanced ratio of linoleic acid to linolenic acid (2:1), tocopherols, phenolic compounds (especially sinapic acid and its derivatives) and sterols (Szydłowska-Czerniak 2013).

Unfortunately, unsaturated fatty acids undergo rapid oxidation and produce a complex mixture of the primary and secondary oxidation products that cause off-flavours and offodours. Moreover, the rate of oil oxidation depends on

Aleksandra Szydłowska-Czerniak olasz@umk.pl

1 Faculty of Chemistry, Nicolaus Copernicus University in Torun, 7 Gagarin Street, 87-100 Torun, Poland amounts of antioxidants and prooxidants, exposure to oxygen and light, temperature and time, which affect oil quality during processing and storage (Wroniak and Rękas 2016). Therefore, the supplementation of oils with antioxidants retards oxidation of unsaturated fatty acids and consequently decreases oils' rancidity. Synthetic antioxidants such as butylated hydroxyanisole (BHA), butylated hydroxytoluene (BHT), propyl gallate (PG) and tert-butylhydroquinone (TBHQ) are commonly used by fat industry due to their chemical stability, low cost, availability and effective prevention of oxidation. However, the toxic and carcinogenic effects of these antioxidants for human health limit their application in food products. For this reason, natural antioxidants extracted from plants, herbs and spices such as celery, rosemary, thyme, bay and sage were added to rapeseed oils in order to protect against oxidation (Maleki et al. 2016; Turan 2014). Unfortunately, natural phenolic antioxidants have a hydrophilic character, which reduces their efficacy in inhibiting oil oxidation. Esterification of phenolic acids with alkyl alcohols can enhance the hydrophobicity and antioxidant activity (AA) of 
the synthesized compounds, namely phenolipids. It is well known that phenolipids can improve the AA and oxidative stability of vegetable oils, as well as extend their shelf life (Catel et al. 2012; Szydłowska-Czerniak and Rabiej 2018).

Moreover, quality of edible oils is significantly affected by the type of packaging materials and their colour, shape and storage conditions such as temperature, time, headspace volume, exposure to light (Martínez et al. 2013; Pristouri et al. 2010; Sanmartin et al. 2018; Wroniak and Rękas 2016). For this reason, different packaging materials (tinplate tin, greenish, amber and clear glass, amber and clear polyethylene terephthalate (PET), clear PET + UV blocker, clear polypropylene (PP) and clear PET covered with aluminium foil) were used to extend the shelf life of oils by providing an inert barrier to external conditions, especially oxygen and light. However, amber glass and amber PET containers ensured comparable quality of cold-pressed rapeseed oil over 12 months of storage (Wroniak and Rękas 2016).

On the other hand, among fluorescent molecules are tocopherols, phenolics and chlorophyll naturally present in vegetable oils, as well as hydrolysis and oxidation products created during technological processes and storage. Therefore, fluorescence spectroscopy is a simple, cost-effective, rapid and non-destructive method for monitoring of oxidation changes in edible oils (Poulli et al. 2009; Sikorska et al. 2008). This technique allows avoiding time-consuming, labour-intensive sample pretreatment procedures and minimizes the use of hazardous chemicals.

However, to the best of our knowledge, there are no reports on the application of synchronous fluorescence technique as a tool to determine the degradation degree of rapeseed oils supplemented with novel antioxidant octyl sinapate (OSA) depending on its concentration, package type and storage conditions.

In this work, synchronous fluorescence (SF) spectroscopy, excitation-emission matrix (EEM) fluorescence spectroscopy and UV-VIS spectroscopy were used to evaluate the impact of addition of a novel lipophilic antioxidant OSA at two concentrations and a well-known synthetic antioxidant BHA on oxidative status and AA of refined rapeseed oils stored in different types of packages and subjected to various storage conditions.

\section{Material and Methods}

\section{Reagents}

All reagents of analytical or HPLC grade were purchased from Sigma-Aldrich (Poznań, Poland). Redistilled water was applied for preparation of all solutions.

A novel lipophilic antioxidant OSA was synthesized by Fischer esterification between the sinapic acid and 1-octanol under acid catalysis, purified and identified according to procedures and analytical methods described in our previous report (Szydłowska-Czerniak and Rabiej 2018).

\section{Samples and Storage Conditions}

The fresh refined rapeseed oil in the original packaging (polypropylene (PP) container) was kindly provided by a local vegetable oil factory.

In the next step, 0.06 and $1.50 \mathrm{~g}$ of synthesized and purified OSA were added to fresh oils $(300 \mathrm{~g})$ and stirred for 5 min to obtain final concentrations of 0.02 and $0.5 \%$, respectively. Also, BHA was introduced directly to rapeseed oil at the legal limit of $0.02 \%$. The fresh refined rapeseed oils without $(\mathrm{RO})$ and with antioxidants $(\mathrm{RO}+$ $0.02 \mathrm{OSA}, \mathrm{RO}+0.5 \mathrm{OSA}$ and $\mathrm{RO}+0.02 \mathrm{BHA}$ ) were labelled as the first batch (I).

Moreover, two types of containers, Duran transparent and amber glass bottles with blue screw caps, $50 \mathrm{~mL}, 46 \times 91 \mathrm{~mm}$, were filled for these studies. All containers (each bottle with $\mathrm{RO}, \mathrm{RO}+0.02 \mathrm{OSA}, \mathrm{RO}+0.5 \mathrm{OSA}$ and $\mathrm{RO}+0.02 \mathrm{BHA}$, respectively) were stored in different places to simulate household and supermarket storage conditions. Oil samples in Duran transparent glass bottles were kept in the following conditions: the second batch (II), in a refrigerator at $4{ }^{\circ} \mathrm{C}$; the third batch (III), at room temperature (the average $T=$ $21{ }^{\circ} \mathrm{C}$ ) in the dark; the fourth batch (IV), exposed to direct light (day-night) in transparent glass bottles; fifth batch (V), exposed to direct light (day-night on the lab shelf) in amber glass bottles. The storage conditions were not controlled during the 6-month studies.

\section{Chemical Analysis of Oil Samples}

The peroxide value (PV) and p-anisidine value (p-AnV) that indicate the primary and secondary oxidation products of oil, respectively, were analysed by the official methods: International Organization for Standardization (2017) and International Organization for Standardization (2016). The oxidation state of oil given by the TOTOX was calculated according to the following formula: $\mathrm{TOTOX}=2 \mathrm{PV}+\mathrm{p}$ AnV.

Extinction coefficients $\left(K_{232}\right.$ and $\left.K_{268}\right)$, expressed as the specific absorptivity values of $1 \%$ solution of oil in n-hexane at 232 and $268 \mathrm{~nm}$, respectively, in 1-cm cell path length (UVVIS spectrophotometer Hitachi U-2900, Tokyo, Japan), were determined according to International Organization for Standardization (2011) method.

The degree of oil hydrolysis was assessed by measuring the acid value (AV) using the official method International Organization for Standardization (1996).

The AA of each rapeseed oil sample was determined by the modified spectrophotometric DPPH method described previously (Szydłowska-Czerniak and Łaszewska 2015). 


\section{Fluorescence Spectroscopy}

Fluorescence spectra were recorded on a fully computerized spectrofluorometer Gilden p $\lambda$ otonics, and a xenon lamp was used as a source for excitation. Each oil sample diluted in nhexane $(1 \%)$ was set in a $10-\mathrm{mm}$ quartz cuvette. All threedimensional spectra were generated by measuring the emission spectra in the range from 250 to $700 \mathrm{~nm}$ repeatedly, at excitation wavelengths from 250 to $450 \mathrm{~nm}$, spaced by $10-\mathrm{nm}$ intervals in the excitation domain. The acquisition interval and the integration time were maintained at $1 \mathrm{~nm}$ and $0.1 \mathrm{~s}$, respectively. Fully corrected spectra were concatenated into an excitation-emission matrix (EEM). The main advantage of EEM is that more information about the fluorescent species can be extracted due to a wider area available for analysis. The intrinsic presence of scatter effects in the EEM landscapes poses a practical problem. Therefore, Rayleigh signals were removed in all EEM by inserting the zero in regions, where $\lambda_{\mathrm{em}} \leq \lambda_{\mathrm{exc}}$ and $\lambda_{\mathrm{em}} \geq 2 \times \lambda_{\mathrm{exc}}$ (Kumar and Mishra 2013).

Synchronous fluorescence (SF) spectra were collected by simultaneously scanning the excitation and emission monochromators. The excitation and emission wavelengths were scanned in the 250-700-nm range with a wavelength resolution of $5 \mathrm{~nm}$ and a constant wavelength difference $(\Delta \lambda)$ between them: 10, 30, 60 and $80 \mathrm{~nm}$. Fluorescence intensities were plotted as a function of the excitation wavelength.

\section{Statistical Analysis}

The oxidation parameters PV, p-AnV, AV, $K_{232}$ and $K_{268}$ for each oil sample were analysed three times within 1 day, while the AA of the non-fortified and fortified rapeseed oils stored in various bottles under different conditions were estimated by fivefold determination of each extract within the same day using the modified DPPH method. The obtained results were presented as follows: mean $(c) \pm$ standard deviation (SD). All data were statistically evaluated by the analysis of variance (ANOVA) test. A post hoc Duncan's test was applied for the calculation of the significant differences among mean values of characteristic oil parameters at the probability level $p<0.05$.

Statistical analysis of data was performed using the Statistica 8.0 software (StatSoft, Tulsa, OK, USA).

\section{Results and Discussion}

\section{Chemical Parameters of the Stored Oils}

Oxidation status of rapeseed oils without and with OSA and BHA after 6 months of storage was evaluated by characteristic parameters. The PV, $K_{232}$ and $K_{268}$ values were used to measure changes in the content of peroxides including conjugated dienes and conjugated trienes, whereas the $\mathrm{p}-\mathrm{AnV}$ value is associated with the formation of secondary oxidation products. However, changes in AV of oils during storage are the result of triacylglycerol breakdown, due to hydrolysis or lipolysis, in which free fatty acids are formed. Moreover, effects of antioxidants added to oils and storage conditions on their antioxidant potential were determined by the modified DPPH method (Table 1).

Amounts of the primary (PV $=0.53 \mathrm{meq} \mathrm{O}_{2} / \mathrm{kg}$ ) and secondary $(\mathrm{p}-\mathrm{AnV}=1.39)$ oxidation products, overall oxidation state (TOTOX $=2.45)$ and acidity $(\mathrm{AV}=0.029 \mathrm{mg} \mathrm{NaOH} / \mathrm{g})$ of fresh rapeseed oil before antioxidant addition were the lowest and below the legal limits (PV $<5$ meq $\mathrm{O}_{2} / \mathrm{kg}$ and $\mathrm{p}-\mathrm{AnV}$ $<8$, TOTOX $<10$ and $\mathrm{AV}<0.300 \mathrm{mg} \mathrm{NaOH} / \mathrm{g}$ ) permitted for refined oils according to International Organization for Standardization $(2017,2016,1996)$. As can be seen, novel antioxidant OSA and BHA caused an insignificant increase in the PV $\left(0.69-0.77\right.$ meq $\left.\mathrm{O}_{2} / \mathrm{kg}\right), K_{232}(2.241-2.299)$ and $K_{268}(0.483-0.525)$ values of the studied oils from batch I (Duncan's test, Table 1).

Also, similar $K_{232}$ results (approximately 2.500 ) for rapeseed oil with celery extract, TBHQ and control sample were observed (Maleki et al. 2016).

However, refined rapeseed oil with studied antioxidants had significantly higher contents of secondary oxidation products $(\mathrm{p}-\mathrm{AnV}=1.96-2.19)$, free fatty acids $\left(\mathrm{AV}=0.038_{-}^{-}\right.$ $0.328 \mathrm{mg} \mathrm{NaOH} / \mathrm{g}$ ) and antioxidant potential ( $\mathrm{DPPH}=625$ $2727 \mu \mathrm{mol} \mathrm{TE} / 100 \mathrm{~g}$ ). A higher $\mathrm{p}-\mathrm{AnV}$ for the fortified rapeseed oils suggests that the intensive mixing of oils with antioxidants during enrichment caused an increase in content of secondary compounds originating from the breakdown of hydroperoxides.

It is evident that the addition of OSA resulted in a higher AV in the initial oils, depending on its concentration (Table 1). This fact confirms acidity of phenolic groups (hydroxyl groups) present in sinapate ester moiety (Musialik et al. 2009). Therefore, OSA added to oil reacts with alkali to form its sodium derivative and contributes significantly to oil's acidity expressed as AV. On the other hand, OSA can be hydrolyzed to yield free acid and alcohol.

For comparison, an increase in AV $(0.33-0.51 \mathrm{mg} \mathrm{KOH} / \mathrm{g})$ of rapeseed oils fortified with sterol oleate, sterol linoleate, sterol linolenate and fat-soluble tea polyphenols in comparison with the non-supplemented oil sample (AV $=0.27 \mathrm{mg}$ $\mathrm{KOH} / \mathrm{g}$ ) was found (Qianchun et al. 2014).

It is noteworthy that the same level $(0.02 \%)$ of the novel antioxidant OSA and BHA caused a significant increase in antioxidant potential of fresh rapeseed oil (33 and $45 \%$, respectively, Table 1). Obviously, the AA of the fortified oils increased by increasing the concentration of added OSA. Therefore, fresh rapeseed oil with 0.02 and $0.5 \%$ of OSA revealed approximately 1.5 and 6.5 times higher DPPH results, respectively, than oil without phenolipid. 
Table 1 Effect of antioxidants, storage conditions and packaging type on rapeseed oil quality

\begin{tabular}{|c|c|c|c|c|c|c|c|}
\hline Oil sample & $\begin{array}{l}\mathrm{PV}^{*} \pm \mathrm{SD} \text { (meq } \\
\left.\mathrm{O}_{2} / \mathrm{kg}\right)\end{array}$ & $\mathrm{p}-\mathrm{AnV}^{*} \pm \mathrm{SD}$ & TOTOX & $K_{232} * \pm \mathrm{SD}$ & $K_{268} * \pm \mathrm{SD}$ & $\begin{array}{l}\mathrm{AV}^{*} \pm \mathrm{SD} \\
(\mathrm{mg} \mathrm{NaOH} / \mathrm{g})\end{array}$ & $\begin{array}{l}\mathrm{DPPH}^{\#} \pm \mathrm{SD}(\mu \mathrm{mol} \\
\mathrm{TE} / 100 \mathrm{~g})\end{array}$ \\
\hline \multicolumn{8}{|c|}{ Batch I-fresh refined rapeseed oil without and with antioxidants } \\
\hline RO & $0.53 \pm 0.02^{\mathrm{a}}$ & $1.39 \pm 0.04^{\mathrm{a}}$ & 2.45 & $2.250 \pm 0.091^{\mathrm{a}, \mathrm{b}}$ & $0.508 \pm 0.008^{\mathrm{a}, \mathrm{b}}$ & $0.029 \pm 0.001^{\mathrm{a}}$ & $421 \pm 12^{\mathrm{c}}$ \\
\hline $\mathrm{RO}+0.02 \mathrm{OSA}$ & $0.77 \pm 0.01^{\mathrm{a}}$ & $2.19 \pm 0.04^{\mathrm{b}, \mathrm{c}}$ & 3.73 & $2.299 \pm 0.026^{\mathrm{a}, \mathrm{b}}$ & $0.525 \pm 0.010^{\mathrm{b}, \mathrm{c}}$ & $0.077 \pm 0.003^{\mathrm{b}, \mathrm{c}, \mathrm{d}}$ & $625 \pm 9^{\mathrm{g}}$ \\
\hline $\mathrm{RO}+0.5 \mathrm{OSA}$ & $0.69 \pm 0.02^{\mathrm{a}}$ & $2.00 \pm 0.04^{\mathrm{b}}$ & 3.38 & $2.280 \pm 0.033^{\mathrm{a}, \mathrm{b}, \mathrm{c}}$ & $0.521 \pm 0.018^{\mathrm{b}, \mathrm{c}}$ & $0.328 \pm 0.006^{\mathrm{g}}$ & $2727 \pm 126^{\mathrm{m}}$ \\
\hline $\mathrm{RO}+0.02 \mathrm{BHA}$ & $0.75 \pm 0.01^{\mathrm{a}}$ & $1.96 \pm 0.08^{\mathrm{b}}$ & 3.46 & $2.241 \pm 0.003^{\mathrm{a}, \mathrm{b}}$ & $0.483 \pm 0.023^{\mathrm{a}}$ & $0.038 \pm 0.001^{\mathrm{a}, \mathrm{b}}$ & $762 \pm 6^{\mathrm{h}}$ \\
\hline \multicolumn{8}{|c|}{ Batch II—refined rapeseed oil without and with antioxidants stored in a refrigerator at $4{ }^{\circ} \mathrm{C}$} \\
\hline RO & $1.71 \pm 0.05^{\mathrm{a}}$ & $2.61 \pm 0.12^{\mathrm{d}}$ & 5.97 & $2.309 \pm 0.013^{\mathrm{a}, \mathrm{b}, \mathrm{c}}$ & $0.576 \pm 0.009^{\mathrm{e}, \mathrm{f}}$ & $0.053 \pm 0.001^{\mathrm{a}, \mathrm{b}, \mathrm{c}}$ & $322 \pm 9^{\mathrm{a}, \mathrm{b}}$ \\
\hline $\mathrm{RO}+0.02 \mathrm{OSA}$ & $1.29 \pm 0.06^{\mathrm{a}}$ & $2.32 \pm 0.05^{\mathrm{c}, \mathrm{d}}$ & 4.90 & $2.291 \pm 0.054^{\mathrm{a}, \mathrm{b}}$ & $0.562 \pm 0.027^{\mathrm{d}, \mathrm{e}}$ & $0.095 \pm 0.004^{\mathrm{d}, \mathrm{e}}$ & $569 \pm 13^{\mathrm{f}}$ \\
\hline $\mathrm{RO}+0.5 \mathrm{OSA}$ & $0.91 \pm 0.01^{\mathrm{a}}$ & $2.19 \pm 0.09^{\mathrm{b}, \mathrm{c}}$ & 4.01 & $2.288 \pm 0.037^{\mathrm{a}, \mathrm{b}}$ & $0.518 \pm 0.022^{\mathrm{b}, \mathrm{c}}$ & $0.367 \pm 0.014^{\mathrm{h}}$ & $2478 \pm 59^{\mathrm{k}}$ \\
\hline $\mathrm{RO}+0.02 \mathrm{BHA}$ & $1.56 \pm 0.07^{\mathrm{a}}$ & $2.55 \pm 0.08^{\mathrm{d}}$ & 5.73 & $2.212 \pm 0.026^{\mathrm{a}}$ & $0.545 \pm 0.014^{\mathrm{c}, \mathrm{d}, \mathrm{e}}$ & $0.041 \pm 0.000^{\mathrm{a}, \mathrm{b}}$ & $464 \pm 20^{\mathrm{c}, \mathrm{d}}$ \\
\hline \multicolumn{8}{|c|}{ Batch III—refined rapeseed oil without and with antioxidants stored at room temperature in the dark } \\
\hline RO & $1.91 \pm 0.04^{\mathrm{a}}$ & $2.94 \pm 0.14^{\mathrm{e}}$ & 6.76 & $2.333 \pm 0.064^{\mathrm{b}, \mathrm{c}, \mathrm{d}}$ & $0.581 \pm 0.015^{\mathrm{d}, \mathrm{e}}$ & $0.073 \pm 0.003^{\mathrm{b}, \mathrm{c}, \mathrm{d}}$ & $335 \pm 9^{\mathrm{b}}$ \\
\hline $\mathrm{RO}+0.02 \mathrm{OSA}$ & $1.26 \pm 0.05^{\mathrm{a}}$ & $2.46 \pm 0.02^{\mathrm{c}, \mathrm{d}}$ & 4.98 & $2.332 \pm 0.076^{\mathrm{b}, \mathrm{c}, \mathrm{d}}$ & $0.560 \pm 0.025^{\mathrm{e}, \mathrm{f}}$ & $0.116 \pm 0.005^{\mathrm{e}, \mathrm{f}}$ & $550 \pm 11^{\mathrm{e}, \mathrm{f}}$ \\
\hline $\mathrm{RO}+0.5 \mathrm{OSA}$ & $1.20 \pm 0.05^{\mathrm{a}}$ & $2.44 \pm 0.03^{\mathrm{c}, \mathrm{d}}$ & 4.84 & $2.301 \pm 0.033^{\mathrm{a}, \mathrm{b}, \mathrm{c}}$ & $0.559 \pm 0.011^{\mathrm{d}, \mathrm{e}}$ & $0.383 \pm 0.007^{\mathrm{h}}$ & $2558 \pm 49^{1}$ \\
\hline $\mathrm{RO}+0.02 \mathrm{BHA}$ & $1.56 \pm 0.03^{\mathrm{a}}$ & $2.22 \pm 0.09^{\mathrm{b}, \mathrm{c}}$ & 5.34 & $2.293 \pm 0.080^{\mathrm{a}, \mathrm{b}}$ & $0.533 \pm 0.020^{\mathrm{b}, \mathrm{c}, \mathrm{d}}$ & $0.032 \pm 0.001^{\mathrm{a}}$ & $508 \pm 21^{\mathrm{d}, \mathrm{e}}$ \\
\hline \multicolumn{8}{|c|}{ Batch IV—refined rapeseed oil without and with antioxidants exposed to direct light in transparent glass bottles } \\
\hline $\mathrm{RO}$ & $59.89 \pm 0.13^{\mathrm{h}}$ & $8.56 \pm 0.23^{\mathrm{j}}$ & 128.34 & $2.586 \pm 0.057^{\mathrm{f}, \mathrm{g}}$ & $0.757 \pm 0.033^{\mathrm{h}}$ & $0.085 \pm 0.004^{\mathrm{c}, \mathrm{d}, \mathrm{e}}$ & $281 \pm 9^{\mathrm{a}}$ \\
\hline $\mathrm{RO}+0.02 \mathrm{OSA}$ & $35.15 \pm 0.21^{\mathrm{f}}$ & $3.77 \pm 0.09^{\mathrm{h}}$ & 74.07 & $2.488 \pm 0.029^{\mathrm{d}, \mathrm{e}, \mathrm{f}}$ & $0.645 \pm 0.021^{\mathrm{f}}$ & $0.142 \pm 0.002^{\mathrm{f}}$ & $473 \pm 13^{\mathrm{c}, \mathrm{d}}$ \\
\hline $\mathrm{RO}+0.5 \mathrm{OSA}$ & $11.22 \pm 0.30^{\mathrm{c}}$ & $3.68 \pm 0.08^{\mathrm{g}, \mathrm{h}}$ & 26.12 & $2.417 \pm 0.043^{\mathrm{c}, \mathrm{d}, \mathrm{e}}$ & $0.602 \pm 0.012^{\mathrm{g}}$ & $0.503 \pm 0.020^{\mathrm{i}}$ & $1687 \pm 56^{\mathrm{i}}$ \\
\hline $\mathrm{RO}+0.02 \mathrm{BHA}$ & $16.80 \pm 0.27^{\mathrm{d}}$ & $3.33 \pm 0.12^{\mathrm{f}}$ & 36.93 & $2.460 \pm 0.013^{\mathrm{e}, \mathrm{f}}$ & $0.575 \pm 0.013^{\mathrm{e}, \mathrm{f}}$ & $0.075 \pm 0.003^{\mathrm{b}, \mathrm{c}, \mathrm{d}}$ & $451 \pm 13^{\mathrm{c}, \mathrm{d}}$ \\
\hline \multicolumn{8}{|c|}{ Batch V-refined rapeseed oil without and with antioxidants exposed to direct light in amber glass bottles } \\
\hline RO & $48.47 \pm 1.17^{\mathrm{g}}$ & $5.97 \pm 0.36^{\mathrm{i}}$ & 102.91 & $2.538 \pm 0.062^{\mathrm{g}}$ & $0.665 \pm 0.032^{\mathrm{g}}$ & $0.074 \pm 0.002^{\mathrm{b}, \mathrm{c}, \mathrm{d}}$ & $293 \pm 13^{\mathrm{a}, \mathrm{b}}$ \\
\hline $\mathrm{RO}+0.02 \mathrm{OSA}$ & $28.88 \pm 0.21^{\mathrm{e}}$ & $3.76 \pm 0.06^{\mathrm{h}}$ & 61.52 & $2.420 \pm 0.023^{\mathrm{d}, \mathrm{e}}$ & $0.654 \pm 0.017^{\mathrm{g}}$ & $0.142 \pm 0.005^{\mathrm{f}}$ & $476 \pm 9^{\mathrm{d}}$ \\
\hline $\mathrm{RO}+0.5 \mathrm{OSA}$ & $7.67 \pm 0.20^{\mathrm{b}}$ & $3.19 \pm 0.05^{\mathrm{e}, \mathrm{f}}$ & 18.53 & $2.316 \pm 0.073^{\mathrm{b}, \mathrm{c}}$ & $0.569 \pm 0.027^{\mathrm{e}, \mathrm{f}}$ & $0.475 \pm 0.005^{\mathrm{i}}$ & $1877 \pm 83^{\mathrm{j}}$ \\
\hline $\mathrm{RO}+0.02 \mathrm{BHA}$ & $9.81 \pm 0.40^{\mathrm{c}}$ & $3.43 \pm 0.12^{\mathrm{f}, \mathrm{g}}$ & 23.05 & $2.396 \pm 0.016^{\mathrm{c}, \mathrm{d}, \mathrm{e}}$ & $0.570 \pm 0.008^{\mathrm{e}, \mathrm{f}}$ & $0.055 \pm 0.002^{\mathrm{a}, \mathrm{b}, \mathrm{c}}$ & $457 \pm 17^{\mathrm{c}, \mathrm{d}}$ \\
\hline
\end{tabular}

Different letters within the same column indicate significant differences between chemical parameters of the studied oils (Duncan's test, $p<0.05$ ) $S D$ standard deviation; $R O$ rapeseed oil; $R O+0.02 O S A$ rapeseed oil with $0.02 \%$ of octyl sinapate; $R O+0.5 O S A$ rapeseed oil with $0.5 \%$ of octyl sinapate; $R O+0.02 B H A$ rapeseed oil with $0.02 \%$ of butylated hydroxyanisole

${ }^{*} n=3 ;{ }^{*} n=5$

As expected, the oxidative parameters of the studied rapeseed oils were the most affected by the packaging and storage conditions, as well as the concentration and composition of additives. The Duncan test indicated that there are insignificant differences in the $\mathrm{PV}$ and $K_{232}$ results between oil samples without and with antioxidants stored at $4{ }^{\circ} \mathrm{C}$ and room temperature in the dark (Table 1). On the other hand, the formation of hydroperoxides and conjugated dienes and trienes was slower in the supplemented oils exposed to direct light for 6 months. The protection effect of the added OSA increased by increasing its concentration in the refined rapeseed oil. Therefore, oil with $0.5 \%$ OSA under the influence of sunlight revealed approximately 3 times lower PV than oil with $0.02 \%$ of OSA. Also, an increase in conjugated dienes and trienes was lower in oils with higher amounts of OSA = $0.5 \%$ than in oils supplemented with $0.02 \%$ of OSA and exposed to UV light. However, the highest concentrations of peroxides $\left(\mathrm{PV}=11.22-59.89 \mathrm{meq} \mathrm{O}_{2} / \mathrm{kg}\right)$, conjugated dienes $\left(K_{232}=2.417-2.586\right)$ and conjugated trienes $\left(K_{268}=0.575-\right.$ 0.757 ) were observed for oils over 6 months of storage in closed transparent glass bottles under UV light exposure. Significantly, lower evolution of the peroxides and conjugated dienes in oils packed in amber glass bottles during the period of analysis was found. Nevertheless, the PV results for all oils exposed to light in amber glass containers (7.67-48.47 meq $\mathrm{O}_{2} / \mathrm{kg}$ ) higher than that recommended by International Organization for Standardization (2017) $\left(\mathrm{PV}<5\right.$ meq $\left.\mathrm{O}_{2} / \mathrm{kg}\right)$ suggest quality degradation in terms of oxidative rancidity. The changes in amounts of hydroperoxides and diene and triene compounds can be expected due to the relatively unstable nature of these molecules generated in oils and their liability to break down into smaller compounds such as aldehydes and ketones. 
Interestingly, the PV (5.96-30.26 meq $\left.\mathrm{O}_{2} / \mathrm{kg}\right), K_{232}(2.10$ $5.50)$ and $K_{268}(0.13-0.23)$ results for 12-month-stored coldpressed rapeseed oils in amber glass and PET containers in the dark at $20^{\circ} \mathrm{C}$ were higher than those exposed to light at the same bottles and temperature ( $\mathrm{PV}=5.72-28.25 \mathrm{meq} \mathrm{O}_{2} / \mathrm{kg}$, $K_{232}=2.05-3.47$ and $K_{268}=0.13-0.21$ ), whereas storage at $4{ }^{\circ} \mathrm{C}$ resulted in the slowest generation of hydroperoxides $\left(\mathrm{PV}=4.77-12.70 \mathrm{meq} \mathrm{O}_{2} / \mathrm{kg}\right)$, conjugated dienes $\left(K_{232}=\right.$ $1.89-2.19)$ and conjugated trienes $\left(K_{268}=0.10-0.11\right)$ in the investigated oils (Wroniak and Rękas 2016). On the other hand, walnut oils stored in transparent glass bottles in darkness $\left(\mathrm{PV}=17.69-68.87 \mathrm{meq} \mathrm{O}_{2} / \mathrm{kg}, K_{232}=3.12-7.87\right.$ and $\left.K_{270}=0.09-0.17\right)$ and under light $(\mathrm{PV}=22.25-70.92 \mathrm{meq}$ $\mathrm{O}_{2} / \mathrm{kg}, K_{232}=2.62-7.36$ and $\left.K_{270}=0.08-0.16\right)$ during 90 and 180 days, without addition of any antioxidant oxidized at a similar rate. However, significant lower contents of hydroperoxides ( $\mathrm{PV}=1.01-54.11 \mathrm{meq} \mathrm{O}_{2} / \mathrm{kg}$ ), conjugated dienes $\left(K_{232}=1.23-6.63\right)$ and conjugated trienes $\left(K_{270}=0.07-\right.$ 0.13 ) were determined in walnut oils enriched with TBHQ, ascorbyl palmitate, rosemary extract and their mixtures and stored in darkness (Martínez et al. 2013).

In contrast, the colour of containers did not affect the content of secondary oxidation products $(\mathrm{p}-\mathrm{AnV}=3.19-3.77)$ in enriched oils stored in the presence of daylight, whereas significantly lower p-AnV (2.19-2.55) had oils stored in the complete absence of light (from a refrigerator and dark place). It is noteworthy that the limit of $\mathrm{p}-\mathrm{AnV}=8$ fixed by International Organization for Standardization (2016) was exceeded only for the refined rapeseed oil from transparent glass bottles ( $\mathrm{p}-\mathrm{AnV}=8.56)$ being the most permeable to UV light after 6 months, and hence, these conditions and lack of exogenous antioxidants facilitate the formation and decomposition of hydroperoxides to secondary oxidation products (Table 1).

The control rapeseed oils without and with antioxidants kept out from direct light in transparent glass bottles had TOTOX between 4.01 and 6.76 (Table 1). Interestingly, oils with $0.5 \%$ of OSA stored in transparent and amber glass bottles under the influence of sunlight at room temperature can be evaluated as acceptable since the TOTOX value was lower than 30 . However, refined rapeseed oil and samples with lower concentration of antioxidants exposed to light during 6 months (except oil with $0.02 \%$ of BHA in amber bottle) had high levels of TOTOX (36.93-128.34).

Furthermore, storage of rapeseed oils without and with antioxidants for 6 months caused a slight increase in their acidity (Table 1). Unexpectedly, the Duncan test indicated insignificant differences in $\mathrm{AV}$ results between refined rapeseed oils and oils with $0.02 \%$ of BHA held under different storage conditions. However, a significant effect of light exposure on free fatty acid content in rapeseed oils enriched with OSA was observed (AV $=0.095-0.116$ and $0.367-0.383 \mathrm{mg}$ $\mathrm{NaOH} / \mathrm{g}$ for oils with 0.02 and $0.5 \%$ of OSA stored in the complete absence of light, as well as AV $=0.142$ and 0.475 $0.503 \mathrm{mg} \mathrm{NaOH} / \mathrm{g}$ for oils with OSA exposed to light). It can be noted that all studied oils supplemented with $0.5 \%$ of OSA had AV (0.328-0.503 $\mathrm{mg} \mathrm{NaOH} / \mathrm{g})$ above the desirable level (AV $<0.300 \mathrm{mg} \mathrm{NaOH} / \mathrm{g}$ ) for refined oil (International Organization for Standardization, 1996). This can be explained by the fact that addition of novel ester OSA probably promotes hydrolysis reactions under storage conditions and increases the formation of free fatty acids in the supplemented rapeseed oils.

For comparison, a similar slight increase in acidity of coldpressed rapeseed oils (AV $=0.59-0.75 \%)$ and extra virgin olive oils $(0.63-0.85 \%)$ stored in different types of packages and subjected to variable storage conditions was reported by other authors (Pristouri et al. 2010; Wroniak and Rękas 2016). However, the smallest increase in acidity after 12 months of storage was determined for rapeseed oils and olive oils kept in the darkness, as well as olive samples packaged in PET + UV and PET covered with aluminium foil at $22^{\circ} \mathrm{C}$ in the presence of light.

The antioxidant effect of the added phenolipid is related to its capacity to inhibit the initiation of free radical processes and/or to interrupt the chain reactions in the propagation of oil's oxidation. For this reason, oxidative degradation of rapeseed oils during storage was also observed by the decrease in their AA (Table 1). The Duncan test indicated a significant decrease of DPPH results $(281-2558 \mu \mathrm{mol} \mathrm{TE} / 100 \mathrm{~g})$ for all studied oil samples after 6 months of storage under different conditions with respect to fresh rapeseed oil before and after supplementation with two antioxidants (DPPH $=421$ $2727 \mu \mathrm{mol} \mathrm{TE} / 100 \mathrm{~g}$ ). This suggests that the packaging and storage conditions affect the degradation of both naturally present and added antioxidants to rapeseed oil.

Refined rapeseed oil without synthetic antioxidants had the lowest but similar DPPH results $(281-335 \mu \mathrm{mol} \mathrm{TE} / 100 \mathrm{~g})$ regardless of storage conditions. Also, the enrichment of oils with BHA caused an approximately $40 \%$ decrease of their antioxidant potential after storage in all studied conditions (Table 1). However, the obtained DPPH results for the rapeseed oils with 0.02 and $0.5 \%$ of OSA suggest that storage in the complete absence of light (in a refrigerator and dark place) caused about a $10 \%$ decrease in their antioxidant potential, whereas a significantly higher decrease (24-38\%) in DPPH of oils with the novel antioxidant took place under room temperature and light conditions. This can be explained by the fact that light treatment was the main factor, which degraded both the endogenous and exogenous antioxidants in rapeseed oil causing the DPPH decrease. However, the AA reduction for oils with $0.5 \%$ of OSA after light exposure was higher (31$38 \%$ ) in comparison to oil that contained $0.02 \%$ of OSA $(24 \%)$ and that is stored under the same conditions. Moreover, these results confirm a potential synergism between natural antioxidants and added phenolipid, which 
protects the bioactive compounds against their oxidative damage during storage conditions.

For comparison, the decreases in AA and phenolic content in extra virgin olive oils after 125 days of storage in tinplate tin and greenish glass containers at 6 and $26^{\circ} \mathrm{C}$ were about 15 and $10 \%$, respectively (Sanmartin et al. 2018). Furthermore, walnut oils fortified with synthetic and natural antioxidants and stored in darkness over the period of 90 and 180 days presented higher radical scavenging capacity $\left(\mathrm{DPPH}_{\mathrm{r}}{ }^{-}=\right.$ $8.54-60.92 \%)$ than the oils exposed to light $\left(\mathrm{DPPH}_{\mathrm{r}}{ }^{-}=\right.$ 10.35-61.16\%). Also, strong effects of antioxidant type and its concentration on antioxidant potential of supplemented walnut oils during storage under various conditions were observed (Martínez et al. 2013). Therefore, walnut oil with $200 \mu \mathrm{g} / \mathrm{g}$ of TBHQ stored in darkness had the highest radical scavenging capacity (the lowest amounts of $\mathrm{DPPH}_{\mathrm{r}}{ }^{\circ}=8.54$ $13.23 \%$ were determined).

\section{Fluorescence Studies of the Stored Oils}

The SF spectroscopy was applied for analysis of fluorescent compounds in rapeseed oils without and with new antioxidant OSA and BHA stored under various conditions. In this technique, both the excitation and emission wavelengths are simultaneously scanned at a constant difference between them. As can be seen, the refined rapeseed oil before addition of synthetic antioxidants had relatively stronger fluorescence at $\Delta \lambda=80$ and $30 \mathrm{~nm}$, while a weaker fluorescence was observed at $\Delta \lambda=10 \mathrm{~nm}$ (Fig. 1a).

The fluorescence maximum attributed to tocopherols and phenolic compounds shifted from 300 to $320 \mathrm{~nm}$ with increasing $\Delta \lambda$ from 10 to $80 \mathrm{~nm}$. However, the differences between the excitation and emission wavelengths $\Delta \lambda$ affect the shape and intensity of synchronous spectra. Therefore, the synchronous spectra for lower $\Delta \lambda=10$ and $30 \mathrm{~nm}$ revealed a weak band with a maximum at about 360 and $340 \mathrm{~nm}$, respectively, which can be attributed to tocopherols (Gu et al. 2019).

It was found that at $\Delta \lambda=30 \mathrm{~nm}$, SF spectra of refined rapeseed oil had better resolved features and the fluorescence maxima changed after storage in various conditions (Fig. 1b). The fluorescence maximum at $300 \mathrm{~nm}$ of refined oil samples gradually decreased after storage under refrigeration conditions and exposure to UV light, whereas the fluorescence signal in the region 330-350 increased for oil kept at $4{ }^{\circ} \mathrm{C}$. Moreover, the peak at $300 \mathrm{~nm}$ of rapeseed oils enriched with $0.02 \%$ of OSA and BHA became much stronger and also evolved to lower intensity values after storage in various conditions. This suggests that storage time and exposure to UV light caused the changes in tocopherol homologues and phenolic profile of refined rapeseed oil, as well as suppressed the AA determined by the DPPH method (Table 1). As a consequence, a high value of the correlation coefficient $(r=0.6337$, $p=0.015)$ between tocopherol intensity and DPPH results indicates that tocopherol content affected the antioxidant potential of oil samples without and with $0.02 \%$ of OSA and BHA stored 6 months in darkness under refrigerated and room temperatures, as well as exposed to light.

Also, a decrease in the fluorescence intensity of tocopherols on spectra of various edible oils with increasing time of their storage and exposure to light was observed by Sikorska et al. $(2004,2008,2019)$.

Interestingly, these original fluorescence intensities related with the tocopherols disappeared when $0.5 \%$ of OSA was added to refined rapeseed oil and a new band at $310 \mathrm{~nm}$ increased during exposure to UV light (Fig. 1b). This suggests that the free fatty acids ( $\mathrm{AV}=0.328-0.503 \mathrm{mg} \mathrm{NaOH} / \mathrm{g}$ ) present in high amounts in oils with $0.5 \%$ of OSA can generate the fluorescence signal and reacted with other oil components forming fluorescent compounds with the maximum at $310 \mathrm{~nm}$. For comparison, a low-intensity band with the maximum at $316 \mathrm{~nm}$ in the spectra of extra virgin olive oils stored under light in clear and green glass bottles for 10 months was identified as the photodecomposition and photooxidation products of chlorophyll or other pigments (Sikorska et al. 2008).

Additionally, a sharp intense band with a maximum at $335 \mathrm{~nm}$ characteristic for the fluorescence of exogenous antioxidants was observed in the synchronous spectra of fortified rapeseed oils with $0.5 \%$ of OSA and $0.02 \%$ of BHA. However, the intensity of this band decreased for oil samples stored under refrigerated temperature and exposed to UV light due to a decrease in the level of total antioxidants (lower DPPH values for supplemented oils after storage). For this reason, a linear (correlation coefficient, $r=0.8653$ ) and significant $(p<0.000001)$ correlation was found between DPPH values of all investigated oils and intensity of the fluorescence peak at $335 \mathrm{~nm}$.

It can be noted that the consumption of the endogenous tocopherols in rapeseed oil was faster in samples with the added novel phenolipid OSA, and the rate of depletion was directly related with the concentration of the exogenous antioxidant (the intense band at $300 \mathrm{~nm}$ was found in the spectra of rapeseed oils with $0.02 \%$ of OSA). On the other hand, OSA added at lower concentration $=0.02 \%$ can inhibit the decay of tocopherol and protect the tocopherol from depletion. Furthermore, sinapate ester as a new antioxidant may regenerate tocopherol by the reduction of tocopheroxyl radicals.

The characteristic peaks between 400 and $500 \mathrm{~nm}$ with low intensity in the SF spectra (Fig. 1b) reflect the differences in contents of oxidation products in the studied oils. This fact indicates that new fluorescent oxidation products were formed in rapeseed oils, independently to the type and amount of added antioxidants, but these components underwent additional degradation during storage at investigated conditions. Therefore, spectra of oils without antioxidants and with $0.02 \%$ of OSA having the highest values of PV, p-AnV, AV, $K_{232}$ 

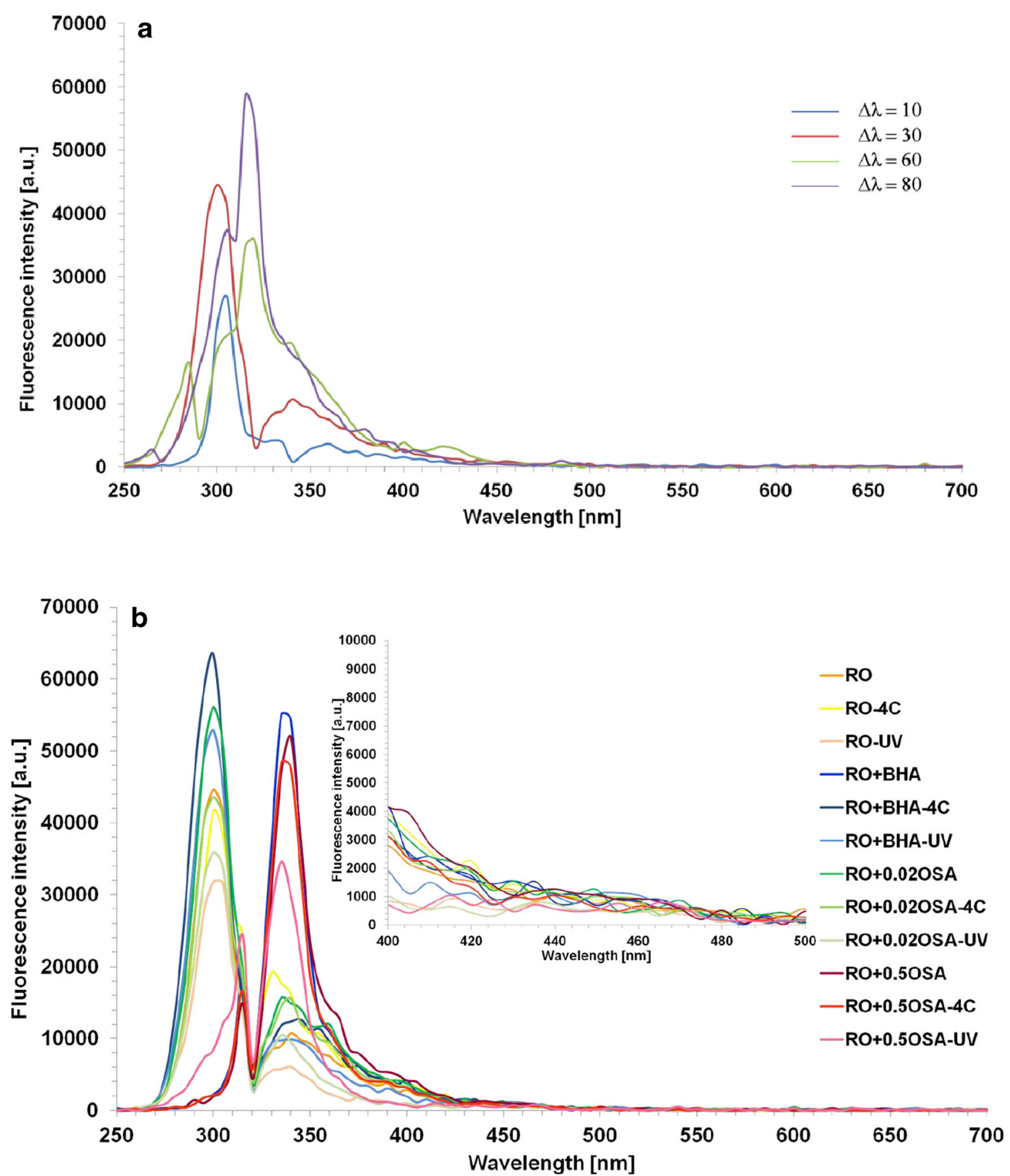

Fig. 1 Synchronous fluorescence spectra of a refined rapeseed oil at different $\Delta \lambda$ and $\mathbf{b}$ refined rapeseed oils without and with antioxidants after storage at various conditions diluted in $\mathrm{n}$-hexane, $c=1 \%$, and recorded at $\Delta \lambda=30$

and $K_{268}$ (Table 1) did not exhibit additional intensities in the 400-500-nm wavelength range.

Nevertheless, SF spectra of all studied rapeseed oils without and with synthetic antioxidants did not reveal fluorescence bands in the range of 550-700 nm, which are attributed to chlorophyll pigments (Fig. 1). This can be explained by the fact that chlorophyll and its derivatives, such as pheophytins and pyropheophytins, were removed from rapeseed oil during refining processes, mainly bleaching. In contrast, the intense fluorescent band between 660 and $700 \mathrm{~nm}$ caused by chlorophyll group pigments was detected in the spectra of mechanically pressed crude rapeseed oils (Sikorska et al. 2004, 2019).

On the other hand, the effect of storage conditions on chemical changes of the main fluorophores in refined rapeseed oils without and with synthetic antioxidants was estimated by two-dimensional excitation-emission matrix (EEM) fluorescence spectroscopy (Fig. 2).

It is evident that the shape and intensity of the fluorescence bands on EEM for fresh refined rapeseed oil and after spiked 
Batch I
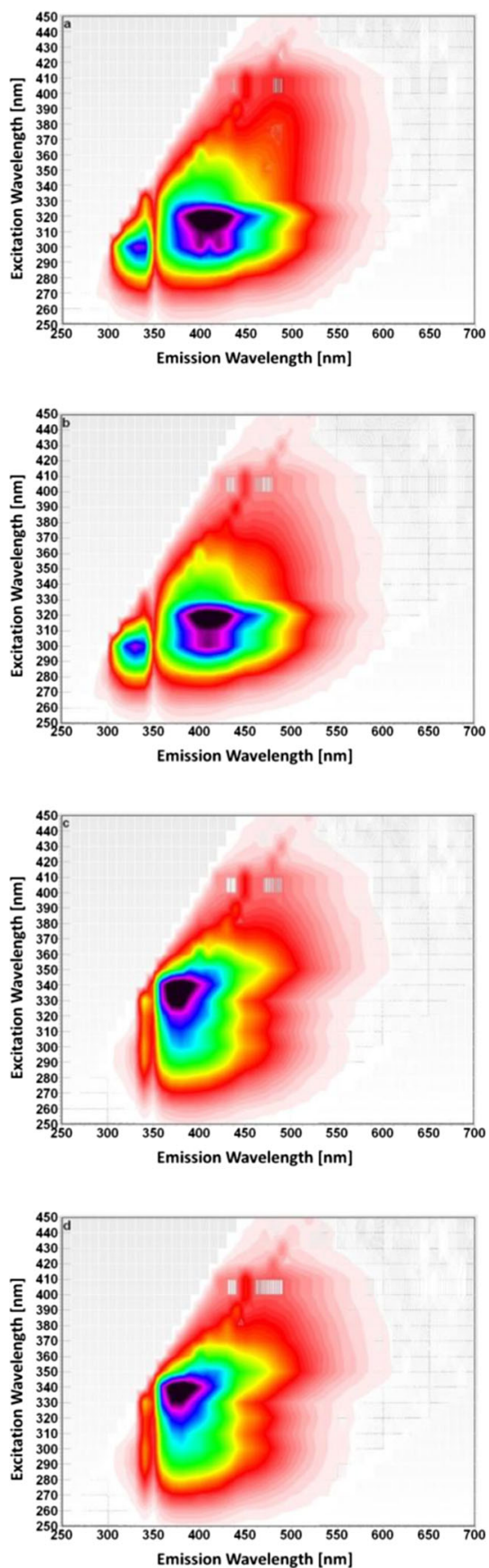

Fig. 2 Excitation-emission matrices for the following: batch I, fresh refined rapeseed oil without (a) and with $0.02 \%$ (b) and $0.5 \%$ (c) of OSA and $0.02 \%$ of BHA (d); batch II, refined rapeseed oil without (e) and with $0.02 \%$ (f) and $0.5 \%$ (g) of OSA and $0.02 \%$ of BHA (h) stored in

with $0.5 \%$ of OSA and $0.02 \%$ of BHA differ significantly (batch I, Fig. 2). The EEM for oils enriched with $0.5 \%$ of OSA and $0.02 \%$ of BHA clearly revealed the greatest emission at $370 \mathrm{~nm}$ with an excitation wavelength of $330 \mathrm{~nm}$ (Fig. $2 \mathrm{c}, \mathrm{d})$. Thus, the newly formed peak at $\lambda_{\text {exc }} / \lambda_{\mathrm{em}}=330 \mathrm{~nm} /$
Batch IV
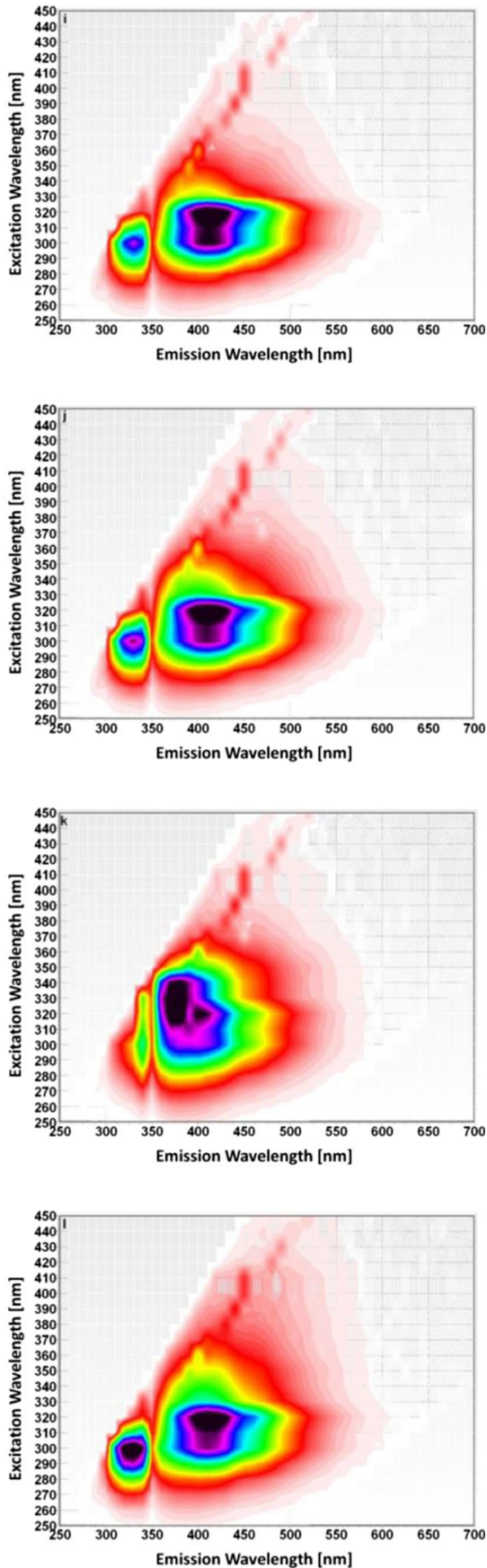

a refrigerator at $4{ }^{\circ} \mathrm{C}$; batch $\mathrm{IV}$, refined rapeseed oil without (i) and with $0.02 \%(\mathbf{j})$ and $0.5 \%(\mathbf{k})$ of OSA and $0.02 \%$ of BHA (l) exposed to direct UV light

$370 \mathrm{~nm}$ indicates a synergistic interaction between tocopherols, phenolics and added phenolipid at higher concentration.

However, the intense fluorescent band in the shortwavelength region $\left(\lambda_{\text {exc }} / \lambda_{\text {em }}=300 / 330 \mathrm{~nm}\right)$ in the refined and supplemented oils with $0.02 \%$ of OSA corresponding to 
tocopherols dramatically decreased after addition of higher amount of new antioxidant and BHA (Fig. 2a-d). Reduction of tocopherol content can be explained by the consequence of the protective effect of the added antioxidants. This relatively intense band corresponding to the naturally present antioxidants (tocopherols and polyphenols) remained almost stable during storage of the refined and enriched rapeseed oils in a refrigerator at $4{ }^{\circ} \mathrm{C}$ (Fig. 2a, b, e, f, h), whereas the exposure to direct UV light caused the degradation of tocopherols and phenolics in oils without exogenous antioxidants and with lower amount of OSA (Fig. 2i, j).

For comparison, the intensities of fluorescence bands ascribed to tocopherols $\left(\lambda_{\mathrm{exc}} / \lambda_{\mathrm{em}}=300 / 331 \mathrm{~nm}\right)$ and pheophytins $\left(\lambda_{\text {exc }} / \lambda_{\text {em }}=400 / 680 \mathrm{~nm}\right)$ decreased considerably in cold-pressed rapeseed oils stored for 6 months in darkness and exposed to light in green and colourless bottles (Sikorska et al. 2019).

Furthermore, the fluorescence intensity of hydroperoxides $\left(\lambda_{\text {exc }} / \lambda_{\text {em }}=320 / 400 \mathrm{~nm}\right)$ present in fresh refined rapeseed oils without and with $0.02 \%$ of OSA (Fig. 2a, b) tended to increase during storage at refrigerated temperature and exposure to UV light (Fig. 2e, f, i, j). However, the intensity of this fluorescence band decreased in oils with $0.5 \%$ of OSA and $0.02 \%$ of BHA stored in a refrigerator (Fig. 2g, h). Changes of this characteristic fluorescence intensity for oil samples without and with antioxidants after storage under various conditions (Fig. 2e-1) can be explained by the fact that primary oxidation products, such as peroxides (PV), conjugated dienes $\left(K_{232}\right)$, conjugated trienes $\left(K_{268}\right)$ and free fatty acids (AV), were degraded and other compounds - secondary oxidation products - such as alcohol, aldehydes and ketones were newly formed by oxidation at the same time. Therefore, rapeseed oils from batch IV exposed to light during 6 months had the highest PV and p-AnV results (Table 1).

\section{Conclusions}

The exogenous antioxidants demonstrated their important influence on rapeseed oil quality during storage under various conditions. The novel amphiphilic antioxidant OSA at higher concentration $(0.5 \%)$ can stabilized rapeseed oil stored over a period of 6 months up to a greater extent than commonly applied synthetic antioxidant BHA. Moreover, radical scavenging activity of rapeseed oil significantly enhanced after supplementation of phenolipid. Rapeseed oils mainly without exogenous antioxidants underwent autooxidation and photosensitized oxidation during the storage, which resulted in a decrease of antioxidant potential and an increase of undesirable component amounts - the primary and secondary oxidation products responsible for nutritional quality.
Therefore, the SF spectra and EEM were applied for a rapid and non-destructive monitoring of the antioxidant effect during the storage of oil samples under various conditions. The formation of new fluorescence products and intensity changes of inherent fluorescent components (tocopherols and phenolic compounds) in rapeseed oil during storage affected the oxidation reactions associated with the deterioration of oils depending on the concentration of added antioxidants, container type, time and conditions.

The synthesized OSA could be used as an efficient antioxidant in the oil industry to delay oxidation reactions and improve the AA of vegetable oils. However, toxicological tests are necessary before proposing use of OSA in food products.

Funding Information Dobrochna Rabiej wishes to thank the Polish National Science Center for the financial support: grant no. 2018/29/N/ NZ9/02748.

\section{Compliance with Ethical Standards}

Conflict of Interest Dobrochna Rabiej declares that she has no conflict of interest. Aleksandra Szydłowska-Czerniak declares that she has no conflict of interest.

Ethical Approval This article does not contain any studies with human or animal subjects.

Informed Consent Informed consent is not applicable in this study.

Open Access This article is licensed under a Creative Commons Attribution 4.0 International License, which permits use, sharing, adaptation, distribution and reproduction in any medium or format, as long as you give appropriate credit to the original author(s) and the source, provide a link to the Creative Commons licence, and indicate if changes were made. The images or other third party material in this article are included in the article's Creative Commons licence, unless indicated otherwise in a credit line to the material. If material is not included in the article's Creative Commons licence and your intended use is not permitted by statutory regulation or exceeds the permitted use, you will need to obtain permission directly from the copyright holder. To view a copy of this licence, visit http://creativecommons.org/licenses/by/4.0/.

\section{References}

Catel Y, Aladedunye F, Przybylski R (2012) Radical scavenging activity and performance of novel phenolic antioxidants in oils during storage and frying. J Am Oil Chem Soc 89:55-66. https://doi.org/10. 1007/s11746-011-1889-6

Gu H, Sun Y, Lv R (2019) A feasibility study for rapid evaluation of oil quality undergoing oven treatment using synchronous fluorescence spectrum. Chem Pap 73:1953-1959. https://doi.org/10.1007/ s11696-019-00748-3

International Organization for Standardization (1996) Animal and vegetable fats and oils - determination of acid value and acidity. ISO 660, Geneva

International Organization for Standardization (2011) Animal and vegetable fats and oils - determination of ultraviolet absorbance expressed as specific UV extinction. ISO 3656, Geneva 
International Organization for Standardization (2016) Animal and vegetable fats and oils - determination of anisidine value. ISO 6885 , Geneva

International Organization for Standardization (2017) Animal and vegetable fats and oils - determination of peroxide value - iodometric (visual) endpoint determination. ISO 3960, Geneva

Kumar K, Mishra AK (2013) Analysis of dilute aqueous multifluorophoric mixtures using excitation-emission matrix fluorescence (EEMF) and total synchronous fluorescence (TSF) spectroscopy: a comparative evaluation. Talanta 117:209-220. https:// doi.org/10.1016/j.talanta.2013.09.002

Maleki M, Ariaii P, Fallah H (2016) Effects of celery extracts on the oxidative stability of canola oil under thermal condition. J Food Process Preserv 40:531-540. https://doi.org/10.1111/jfpp.12632

Martínez ML, Penci MC, Ixtaina V, Ribotta PD, Maestri D (2013) Effect of natural and synthetic antioxidants on the oxidative stability of walnut oil under different storage conditions. LWT-Food Sci Technol 51:44-50. https://doi.org/10.1016/j.lwt.2012.10.021

Musialik M, Kuzmicz R, Pawłowski TS, Litwinienko G (2009) Acidity of hydroxyl groups: an overlooked influence on antiradical properties of flavonoids. J Org Chem 74:2699-2709. https://doi.org/10. $1021 /$ jo802716v

Poulli KI, Chantzos NV, Mousdis GA, Georgiou CA (2009) Synchronous fluorescence spectroscopy: tool for monitoring thermally stressed edible oils. J Agric Food Chem 57:8194-8201. https://doi.org/10.1021/jf902758d

Pristouri G, Badeka A, Kontominas MG (2010) Effect of packaging material headspace, oxygen and light transmission, temperature and storage time on quality characteristics of extra virgin olive oil. Food Control 21:412-418. https://doi.org/10.1016/j.foodcont.2009. 06.019

Qianchun D, Jie S, Mingming Z, Jiqu X, Chuyun W, Qingde H, Qi Z, Pingmei G, Fenghong H, Lan W, Hui T (2014) Thermal stability of rapeseed oil fortified with unsaturated fatty acid sterol esters. J Am Oil Chem Soc 91:1793-1803. https://doi.org/10.1007/s11746-0142527-x

Sanmartin C, Venturi F, Sgherri C, Nari A, Macaluso M, Flamini G, Quartacci MF, Taglieri I, Andrich G, Zinnai A (2018) The effects of packaging and storage temperature on the shelf-life of extra virgin olive oil. Heliyon 4:e00888. https://doi.org/10.1016/j.heliyon.2018. e00888

Sikorska E, Romaniuk A, Khmelinskii IV, Herance R, Bourdelande JL, Sikorski M, Koziol J (2004) Characterization of edible oils using total luminescence spectroscopy. J Fluoresc 14:25-35. https://doi. org/10.1023/B:JOFL.0000014656.75245.62

Sikorska E, Khmelinskii IV, Sikorski M, Caponio F, Bilancia MT, Pasqualone A, Gomes T (2008) Fluorescence spectroscopy in monitoring of extra virgin olive oil during storage. Int J Food Sci Technol 43:52-61. https://doi.org/10.1111/j.1365-2621.2006. 01384.x

Sikorska E, Wójcicki K, Kozak W, Gliszczyńska-Świgło A, Khmelinskii I, Górecki T, Caponio F, Paradiso VM, Summo C, Pasqualone A (2019) Front-face fluorescence spectroscopy and chemometrics for quality control of cold-pressed rapeseed oil during storage. Foods 8 : 1-16. https://doi.org/10.3390/foods 8120665

Szydłowska-Czerniak A (2013) Rapeseed and its products - sources of bioactive compounds: a review of their characteristics and analysis. Crit Rev Food Sci Nutr 53:307-330. https://doi.org/10.1080/ 10408398.2010.529959

Szydłowska-Czerniak A, Łaszewska A (2015) Effect of refining process on antioxidant capacity, total phenolics and prooxidants contents in rapeseed oils. LWT-Food Sci Technol 64:853-859. https://doi.org/ 10.1016/j.lwt.2015.06.069

Szydłowska-Czerniak A, Rabiej D (2018) Octyl sinapate as a new antioxidant to improve oxidative stability and antioxidant activity of rapeseed oil during accelerated storage. Eur Food Res Technol 244:1397-1406. https://doi.org/10.1007/s00217-018-3053-0

Turan S (2014) Effects of some plant extracts on the oxidative stability of canola oil and its purified triacylglycerols. J Food Qual 37:247-258. https://doi.org/10.1111/jfq.12086

Wroniak M, Rękas A (2016) Nutritional value of cold-pressed rapeseed oil during long term storage as influenced by the type of packaging material, exposure to light \& oxygen and storage temperature. J Food Sci Technol 53:1338-1347. https://doi.org/10.1007/s13197015-2082-y

Publisher's Note Springer Nature remains neutral with regard to jurisdictional claims in published maps and institutional affiliations. 